Ergod. Th. \& Dynam. Sys. (1987), 7, 161-164

Printed in Great Britain

\title{
Orbit types for maps of the interval
}

\author{
LOUIS BLOCK AND DAVID HART
}

Department of Mathematics, University of Florida, Gainesville, Florida 32611, USA; Department of Mathematics, University of Cincinnati, Cincinnati, Ohio 45267, USA

(Received 18 June 1986 and revised 26 September 1986)

Abstract. Relationships between the permutations associated to periodic orbits of maps of the interval and the topology of the space of these maps are given. These extend the author's previous work on periodic orbits to orbit types.

Let $I$ denote a compact interval on the real line, and for each positive integer $n$, let $F(n)$ denote the set of $f$ in $C^{0}(I, I)$ such that $f$ has a periodic point of (least) period $n$. A theorem of Šarkovskii [8] asserts that

$$
F(3) \subset F(5) \subset \cdots \subset F(2 \cdot 3) \subset F(2 \cdot 5) \subset \cdots \subset F(4) \subset F(2) \subset F(1) .
$$

Furthermore, if $i \neq j$ and $F(i) \subset F(j)$, then $F(i) \subset$ int $(F(j))$ [3], and if $k$ is not a power two, then $F(k) \cap C^{1}(I, I)$ is a closed subset of $C^{1}(I, I)$ [5]. In general, if $i \neq j$ and $F(i) \subset F(j)$, then the closure of $F(i) \cap C^{1}(I, I)$ is a subset of the interior of $F(j) \cap C^{1}(I, I)[6]$. Thus, the sets $F(k)$ form a stratification of $C^{1}(I, I)$ with strata $F(3), F(5)-F(3)$, and so on. Any $C^{1}$-continuous family of maps $f_{s}$ moving from one of the strata to another must pass through all the strata in between. New periods are created one at a time, in the Šarkovskii order.

However, inside one of the strata, for example $F(5)-F(3)$, many other bifurcations occur. In this paper we see that at least some of these occur in a predictable pattern, which can be described in terms of orbit types of periodic orbits and which refines the Šarkovskii ordering.

We will let $C_{n}$ denote the set of cyclic permutations of $\{1, \ldots, n\}$. An orbit type is just an element of $C_{n}$ for some positive integer $n$. If $X=\left\{x_{1}<x_{2}<\cdots<x_{n}\right\}$ is a periodic orbit of $f \in C^{0}(I, I)$, the orbit type of $X$ is the permutation $F$ defined by $F(i)=j$ if and only if $f\left(x_{i}\right)=x_{j}$.

If $p$ is an orbit type we let $F(p)$ denote the set of maps in $C^{0}(I, I)$ which have a periodic orbit of type $p$. Unlike the Šarkovskii sets, the sets $F(p)$ are not linearly ordered by inclusion. However, in this paper, we show that any linearly ordered sublattice induces a stratification. More precisely, we prove the following.

THEOREM. If $p$ and $q$ are orbit types, with $F(p) \subset F(q)$ and $p \neq q$, then $F(p) \subset$ int $(F(q))$, and

$$
\overline{F(p) \cap C^{1}(I, I)} \subset \operatorname{int}\left(F(q) \cap C^{1}(I, I)\right) .
$$

As noted in [1], it follows from the kneading theory, [7], that the class of unimodal 
orbit types is linearly ordered. Thus, the theorem implies that this class yields a stratification of $C^{1}(I, I)$. This stratification refines the Sarkovskii stratification, in the sense that we may identify the Sarkovskii set $F(n)$ with the set $F(p)$, where $p$ is a 'simple' ([2], [6], [9]) unimodal orbit type of period $n$. Of course, the theorem applies as well to orbit types which are not unimodal.

The main step in obtaining the desired theorem is the following. The remaining step is Theorem 5, below.

THEOREM 1. If $p$ and $q$ are orbit types, with $F(p) \subset F(q)$ and $p \neq q$, then $F(p) \subset$ int $(F(q))$.

The proof of Theorem 1 relies on ideas from [1] and [4] concerning the relationship between loops in the Markov graph and periodic orbits. Suppose $f \in C^{0}(I, I)$ and $X=\left\{x_{1}, \ldots, x_{n}\right\}$ is a periodic orbit of $f$ with $x_{1}<x_{2}<\cdots<x_{n}$. Let $I_{1}=\left[x_{1}, x_{2}\right], \ldots$, $I_{n-1}=\left[x_{n-1}, x_{n}\right]$. The Markov graph of $X$ is the graph with vertices $I_{1}, \ldots, I_{n-1}$ and an arrow from $I_{i}$ to $I_{j}$ if and only if $f\left(I_{i}\right) \supset I_{j}$. If $p$ is an orbit type, the Markov graph of $p$ is the Markov graph of a map $f_{p}$ with an orbit $X=\left\{x_{1}, \ldots, x_{n}\right\}$ of type $p$ such that $f_{p}$ is linear on each interval joining adjacent points of $X$. Given a loop in the Markov graph of $p$, the type of the loop may be obtained (as in [1]). Baldwin proves in [1] that given two orbit types $p$ and $q$ with $p \neq q, F(p) \subset F(q)$ if and only if the Markov graph of $p$ has a non-repetitive loop of type $q$. We will use this result and also part of Baldwin's proof. We first note the following lemma.

Lemma 2. Let $f \in C^{0}(I, I)$ and let $m$ be a positive integer. Let $K=[c, d]$ be a closed subinterval of $I$ such that the intervals $K, f(K), \ldots, f^{m-1}(K)$ are pairwise disjoint. Suppose that at least one of the following holds:

(A) $K \subset$ int $\left(f^{m}(K)\right)$.

(B) $f^{m}(c)>c$ and $f^{m}(d)<d$.

Let $q \in C_{m}$ be the permutation defined by labelling the disjoint intervals $K, \ldots, f^{m-1}(K)$ as $L_{1}, \ldots, L_{m}$ with $L_{1}<L_{2}<\cdots<L_{m}$ and setting $q(i)=j$ iff $\left(L_{i}\right)=L_{j}$ or $L_{i}=f^{m-1}(K)$ and $L_{j}=K$. Then $f \in$ int $(F(q))$.

Proof. The hypothesis of the lemma holds for all $g$ in a neighbourhood of $f$ and implies the existence of a periodic orbit of type $q$.

Proof of Theorem 1. Suppose $p \in C_{n}$ and $q \in C_{m}$. Let $f \in F(p)$, and let $X=\left\{x_{1}, \ldots, x_{n}\right\}$ be a periodic orbit of type $p$. By the theorem of Baldwin, there is a non-repetitive loop

$$
I_{a_{1}} \rightarrow I_{a_{2}} \rightarrow \cdots \rightarrow I_{a_{m}} \rightarrow I_{a_{1}}
$$

in the graph of $X$ which has type $q$. As in the proof of Theorem 3.3 of [1] we can form closed intervals $J_{2 m}, J_{2 m-1}, \ldots, J_{1}, J_{0}$ such that the following hold:

(i) $J_{2 m}=I_{a_{m}}, J_{2 m-1} \subset I_{a_{m-1}}, \ldots, J_{m+1} \subset I_{1}$;

$J_{m} \subset J_{2 m}, J_{m-1} \subset J_{2 m-1}, \ldots, J_{1} \subset J_{m+1} ; J_{0} \subset J_{m}$.

(ii) $f\left(\right.$ int $\left.\left(J_{i}\right)\right)=$ int $\left(J_{i+1}\right)$ for $i=0, \ldots, 2 m-1$.

(iii) $J_{0}, J_{1}, \ldots, J_{m-1}$ are pairwise disjoint.

(iv) If $z$ is a periodic point of $f$ in $J_{0}$ of period $m$, then the orbit of $z$ has type $q$. We have two cases. 
Case 1. $J_{0} \subset \operatorname{int}\left(I_{a_{m}}\right)$.

Since each endpoint of $J_{0}$ is mapped by $f^{2 m}$ to an endpoint of $I_{a_{m}}$, neither endpoint of $J_{0}$ is a fixed point of $f^{m}$. Also, the endpoints of $J_{0}$ are mapped by $f^{m}$ onto the endpoints of $J_{m}$, and $J_{0} \subset J_{m}$. Thus, if $K=J_{0}$, the hypothesis of Lemma 2 is satisfied. Hence, $f \in$ int $(F(q))$.

Case 2. $J_{0}$ is not a subset of int $\left(I_{a_{m}}\right)$.

In this case, each of the intervals $J_{0}, J_{1}, \ldots, J_{m-1}$ contains a point of $X$. Thus, if $m=n$, then $p=q$, a contradiction. Hence, $m \neq n$.

We will show that $m$ is not a multiple of $n$. Suppose $m$ is a multiple of $n$. Then $m \geq 2 n$. It follows that three of the intervals $J_{0}, J_{1}, \ldots, J_{m-1}$ are in the same $I_{j}$. Hence for some $k=0, \ldots, m-1, J_{k}$ contains no elements of $X$. It follows that $J_{0}$ contains no elements of $X$, a contradiction to Case 2. Thus, $m$ is not a multiple of $n$.

Let $J_{0}=[c, d]$. Either $c$ or $d$ is an endpoint of $I_{a_{m}}$; we may suppose without loss of generality that $c$ is. Then $c \in X$, and hence, also $f^{m}(c) \in X$. Since $f^{m}(c) \neq c$, it follows that $f^{m}$ reverses the order of the endpoints of $J_{0}$. Thus, if $K=J_{0}$, the hypothesis of Lemma 2 is satisfied, with (B) holding. Hence, $f \in$ int $(F(q))$, and the proof of Theorem 1 is complete.

Definition. Let $p \in C_{n}$ and $q \in C_{k}$. We say $q=p / 2$ if the following hold:

(i) $k=n / 2$.

(ii) If $\beta_{1}=\{1,2\}, \beta_{2}=\{3,4\}, \ldots, \beta_{k}=\{n-1, n\}$, then $p\left(\beta_{i}\right)=\beta_{j}$ if and only if $q(i)=j$.

The case $q=p / 2$ corresponds to an orbit of type $p$ formed from an orbit of type $q$ by period doubling.

Leмma 3. Suppose $p$ and $q$ are orbit types and $q=p / 2$. Then

$$
\overline{F(p) \cap C^{1}(I, I)} \subset \operatorname{int}\left(F(q) \cap C^{1}(I, I)\right) \text {. }
$$

Proof. Suppose $f$ is the limit of a sequence of maps in $F(p) \cap C^{1}(I, I)$. By Theorem 1 of [5], $f$ has a periodic orbit of type $p$ or $q$. By Theorem 1, we need only consider the case where $f$ has an orbit $X$ of type $q$. In this case, the derivative of $f^{k}$ must be less than or equal to zero at each point of $X$. In particular, the derivative of $f^{k}$ is not equal to one at each point of $X$. Hence, the orbit is preserved under perturbations, and thus, $f \in$ int $\left(F(q) \cap C^{1}(I, I)\right)$.

The following lemma was observed by Baldwin, [1].

Lemma 4. Suppose $p, q$, and $r$ are orbit types with $q=p / 2, p \neq r$, and $F(p) \subset F(r)$. Then $F(q) \subset F(r)$.

From Lemma 3 and its proof, Lemma 4, and Theorem 1, we immediately obtain the following.

THEOREM 5. If $p$ and $q$ are orbit types with $F(p) \subset F(q)$ and $p \neq q$, then

$$
\overline{F(p) \cap C^{1}(I, I)} \subset \text { int }\left(F(q) \cap C^{1}(I, I)\right) \text {. }
$$

We conclude with an intermediate value type result concerning orbit types and families of maps. 
Corollary 6. Suppose $f_{t}$ is a continuous one-parameter family of $C^{1}$ maps of the interval I for $0 \leq t \leq 1$ (i.e. the map $t \rightarrow f_{t}$ from $[0,1]$ to $C^{1}(I, I)$ is continuous). Suppose $p, r$, and $q$ are orbit types with $F(p) \subset F(r) \subset F(q)$ and $r \neq q$. If $f_{1} \in F(p)$ and $f_{0} \notin F(r)$ then there is a parameter $t_{1}$ with $f_{t_{1}} \in F(q)-F(r)$.

Proof. Let $t_{0}$ denote the infimum of $\left\{t \in[0,1]: f_{t} \in F(r)\right\}$. If $f_{t_{0}} \in F(r)$, then for $t_{1}$ slightly less than $t_{0}, f_{t_{1}} \in F(q)-F(r)$. If $f_{t_{0}} \notin F(r)$, then for $t_{1}=t_{0}, f_{t_{1}} \in F(q)-F(r)$.

\section{REFERENCES}

[1] S. Baldwin. Generalizations of a theorem of Šarkovskii on orbits of continuous real-valued functions. Preprint, Auburn University.

[2] L. Block. Simple periodic orbits of mappings of the interval. Trans. Amer. Math. Soc. 254 (1979), 391-398.

[3] L. Block. Stability of periodic orbits in the theorem of Šarkovskii. Proc. Amer. Math. Soc. 81 (1981), 333-336.

[4] L. Block, J. Guckenheimer, M. Misiurewicz \& L.-S. Young. Periodic points and topological entropy of one-dimensional maps. Lecture Notes in Math. 819 (Springer, Berlin, 1980), pp. 18-34.

[5] L. Block \& D. Hart. The bifurcation of periodic orbits of one-dimensional maps. Ergod. Th. \& Dynam. Sys. 2 (1982), 125-129.

[6] L. Block \& D. Hart. Stratification of the space of unimodal interval maps. Ergod. Th. \& Dynam. Sys. 3 (1983), 533-539.

[7] P. Collet \& J-P. Eckmann. Iterated Maps of the Interval as Dynamical Systems. Birkhäuser, Boston, 1980.

[8] A. N. Šarkovskii. Coexistence of cycles of a continuous mapping of the line into itself (Russian). Ukrain. Mat. Ż. 16 (1964), 61-71.

[9] P. Śtefan. A theorem of Šarkovskii on the existence of periodic orbits of continuous endomorphisms of the real line. Comm. Math. Phys. 54 (1977), 237-248. 